\title{
Dynamical Structure and Definition of Energy in General Relativity* $\dagger$
}

R. ARnowitT, Department of Physics, Syracuse University, Syracuse, New York

S. DEser, Department of Physics, Brandeis University, Waltham, Massachusetts

AND

C. W. Misner $\ddagger$ Palmer Physical Laboratory, Princeton University, Princeton, New Jersey

(Received July 6, 1959)

\begin{abstract}
The problem of the dynamical structure and definition of energy for the classical general theory of relativity is considered on a formal level. As in a previous paper, the technique used is the Schwinger action principle. Starting with the full Einstein Lagrangian in first order Palatini form, an action integral is derived in which the algebraic constraint variables have been eliminated. This action possesses a "Hamiltonian" density which, however, vanishes due to the differential constraints. If the differential constraints are then substituted into the action, the true, nonvanishing Hamiltonian of the theory emerges. From an analysis of the equations of motion and the constraint equations, the two pairs of dynamical variables which represent the two independent degrees of freedom of the gravitational field are explicitly exhibited. Four other variables remain in theory; these may be arbitrarily specified, any such specification representing a choice of coordinate frame. It is shown that it is possible to obtain truly canonical pairs of variables in terms of the dynamical and arbitrary variables. Thus a statement of the dynamics is meaningful only after a set of coordinate conditions have been chosen. In general, the true Hamiltonian will be time dependent even for an isolated gravitational field. There thus arises the notion of a preferred coordinate frame, i.e., that frame in which the Hamiltonian is conserved. In this special frame, on physical grounds, the Hamiltonian may be taken to define the energy of the field. In these respects the situation in general relativity is analogous to the parametric form of Hamilton's principle in particle mechanics.
\end{abstract}

\section{INTRODUCTION}

$\mathbf{I}^{\mathrm{N}}$ $\mathrm{N}$ the program of the quantization of the general theory of relativity, ${ }^{1}$ stress has been laid on the necessity of treating the gravitational field as a dynamical system expressed in canonical form. Only when a theory has been expressed in the standard Hamiltonian form arising in Lorentz-covariant theories can the possibility of consistent quantization (by way of the Schwinger action principle) be examined. That this is as feasible for the gravitational field as for the more usual fields is masked by the general coordinate invariance of the theory. In I, a beginning was made by separating out the gauges from the dynamical properties for the linearized approximation; we shall here examine this point for the full classical theory, and indicate formally how the canonical structure is to be reached there. In a subsequent paper, its explicit form will be discussed.

In I, the formulation of the general theory in terms of the action principle was given. In accordance with the basic requirements of the principle, the Lagrangian was stated in first order form with the metric tensor and affinity treated as independent variables. The action principle yields in general three items: the first order Lagrange equations of motion, and two gener-

\footnotetext{
* Supported in part by a National Science Foundation research grant.

$\dagger$ Participation in this research supported in part by the Office of Naval Research.

$\ddagger$ Alfred P. Sloan Research Fellow.

1 R. Arnowitt and S. Deser, Phys. Rev. 113, 745 (1959). This paper will be referred to as I. We use, as in I, natural units: $c=1, \kappa=16 \pi \gamma c^{-4}=1$ ( $\gamma$ is the Newtonian gravitational constant). Greek indices run from 0 to 3 , Latin indices from 1 to 3 , and $x^{0}=t$. Ordinary differentiation is denoted by a comma in a subscript or the symbol $\partial_{\mu}$.
}

ating functions. One generating function gives rise to canonical commutation relations, while the second, generating space-time translations, yields the Heisenberg equations of motion. The requirement that the Heisenberg and Lagrange equations be equivalent varifies the consistency of the quantization procedure.

In general relativity the difficulty in carrying out the above program resides in the invariance under the function group of coordinate transformations. In I, this difficulty was overcome for the linearlized theory. It was seen there that the process of obtaining the correct canonical variables involved making a "gauge" (i.e., linearized coordinate) transformation from an arbitrary gauge to a "radiation" gauge. In this paper we shall extend the analysis to consider the full theory in this light. Of the two types of constraints mentioned in I, the algebraic constraint variables can be handled quite simply in this formalism. At the beginning of the next section their explicit elimination will be carried out. In the process certain combinations of the remaining variables appear in the equations of motion. These combinations remain redundant until the differential constraints are utilized. However, they are physically significant in that the specification of the fields on a given spacelike surface can be given in terms of these combinations. From these considerations it is suggestive to restate the theory in terms of variables that possess the geometrical properties of decomposing the four dimensional characterizations of the space into $3+1$ dimensional aspects. Upon doing this, both the equations of motion and the Lagranian greatly simplify.

The simplified form of the Lagrangian yields a corresponding set of generating functions. The "energymomentum" vector so obtained vanishes identically in 
virtue of the differential constraint equations. Physically, the origin of this phonomenon is related to the general convariance of the theory. This does not mean that the physical energy and momentum of the gravitational field is zero. Further, a stress tensor and hence the energy-momentum vector have meaning only when defined in terms of a specific frame (where definite coordinate conditions have been chosen). This will be seen in detail below. However, the energy so defined will be different in different coordinate frames for the same physical situation. The true energy of the system is that defined in the "radiation" gauge where the Hamiltonian depends only on canonical variables. This follows from another distinguishing characteristic of the Hamiltonian that represents the true energy and of the coordinate frame that is the radiation gauge. Namely, the Hamiltonian should not be an explicit function of the time coordinate since for the free gravitational field, which is a closed system, the energymomentum vector cannot but be conserved if the time coordinate is to be in accord with conventional definitions. Of course, other choices of the coordinate system could be made in which the Hamiltonian does depend explicitly upon the coordinates. While this is logically feasible, the "time" so defined would not match on correctly to the time as employed in the rest of physics, in terms of which closed systems are conservative.

\section{STRUCTURE OF THE EQUATIONS OF MOTION}

In the linearized approximation of the full theory, it was found that the proper dynamical variables were certain combinations of the original variables, $\mathfrak{g}^{\mu \nu}$ and $\Gamma_{\mu \nu}^{\alpha}$, the contravariant metric tensor density and the affinity, respectively. The first step in finding the dynamical variables consisted in the linear theory of eliminating the algebraic constraint variables; the nonlinearity of the full theory does not intrinsically complicate this part of the analysis. We begin with the first order form of the Lagrangian, as stated in I,

$$
\begin{aligned}
I & =\int d^{4} x \mathrm{~g}^{\mu \nu} \cdot R_{\mu \nu}, \\
R_{\mu \nu} & =\Gamma_{\mu \nu, \alpha}^{\alpha}-\frac{1}{2}\left(\Gamma_{\alpha \mu \nu, \nu}^{\alpha}+\Gamma_{\alpha \nu, \mu}^{\alpha}\right) \\
& \quad+\Gamma^{\beta}{ }_{\alpha \beta} \cdot \Gamma_{\mu \nu}^{\alpha}-\Gamma_{\alpha \mu}^{\beta} \cdot \Delta_{\beta \nu}^{\alpha},
\end{aligned}
$$

and $A \cdot B=\frac{1}{2}(A B+B A)$ embodies a symmetrization needed for the quantum case. In this paper we will deal only with the classical problem and hence commutation questions do not enter. The field equations which stem from the independent variation of $\mathrm{g}^{\mu \nu}$ and $\Gamma^{\alpha}{ }_{\mu \nu}$ can be cast in the form

$$
\begin{gathered}
g_{\mu \nu ; \alpha} \equiv g_{\mu \nu, \alpha}-g_{\mu \beta} \Gamma^{\beta}{ }_{\nu \alpha}-g_{\nu \beta} \Gamma^{\beta}{ }_{\mu \alpha}=0, \\
R_{\mu \nu}\left(\Gamma^{\alpha}{ }_{\rho \sigma}\right)=0 .
\end{gathered}
$$

The algebraic constraints are contained in Eq. (2.2a) while Eq. (2.2b) contains the differential constraints. Of the forty equations in (2.2a), thirty are independent of time derivatives. Thus thirty components of the affinity, i.e., $\Gamma^{j}{ }_{i k}, \Gamma^{k}{ }_{i 0}$, and $\Gamma^{0}{ }_{0 k}$ can be eliminated in terms of $\Gamma_{i j}^{0}, \Gamma^{\mu}{ }_{00}$ and the metric. One finds, by manipulations similar to those in I, that

$$
\begin{aligned}
& \Gamma^{j_{i k}}=\frac{1}{2}\left[g^{j m}-\left(g^{00}\right)^{-1} g^{j 0} g^{m 0}\right]\left(g_{i m, k}+g_{m k, i}-g_{i k, m}\right) \\
& +\left(g^{00}\right)^{-1} g^{j 0} \Gamma_{i k}^{0}, \\
& \Gamma^{k}{ }_{i 0}=g^{k m}\left[g_{0 m, i}-\left(g^{00}\right)^{-1} \Gamma_{i m^{0}}\right]+\frac{1}{2} g^{0 k} g_{00, i}+\frac{1}{2}\left(g^{00}\right)^{-1} \\
& \times g^{k n} g^{0 m}\left(g_{i m, n}+g_{n m, i}-g_{i n, m}\right) \text {, } \\
& \Gamma_{0 k}^{0}=g^{0 m}\left[g_{0 m, k}-\left(g^{00}\right)^{-1} \Gamma_{m k}^{0}\right]+\frac{1}{2} g^{00} g_{00, k} \\
& +\left(g^{00}\right)^{-1} g^{0 n} g^{0 m} g_{n m, k} \text {. }
\end{aligned}
$$

The remaining ten equations involve time derivatives and read

where

$$
\begin{aligned}
& \partial_{0} g_{i j}=\Gamma_{i 0 j}+\Gamma_{j 0 i}, \\
& \partial_{0} g_{\mu 0}=\Gamma_{\mu 00}+\Gamma_{00 \mu},
\end{aligned}
$$

$$
\Gamma_{\mu \nu \alpha} \equiv g_{\alpha \beta} \Gamma^{\beta}{ }_{\mu \nu} .
$$

Equations (2.4a) represent dynamical equations for the $\operatorname{six} g_{i j}$, i.e., the spacelike components of the metric tensor. Equations (2.4b) apparently represent dynamical equations for the four $g_{\mu 0}$. However, as in the linearized theory, Eqs. (2.2b) do not yield canonically conjugate equations and hence Eqs. (2.4b) are really defining equations for $\Gamma^{\mu}{ }_{00}$. The absence of equations canonically conjugate to $(2.4 \mathrm{~b})$ arises due to the gauge (i.e., coordinate) invariance of the theory. Thus, the four missing canonically conjugate equations are replaced by the four differential constraint equations implicit in (2.2b). Writing out Eq. (2.2b) explicitly, one has

$$
\begin{aligned}
& \partial_{0} \Gamma^{0}{ }_{i j}=\frac{1}{2}\left(-2 \Gamma^{m}{ }_{i j, m}+\Gamma^{\alpha}{ }_{j \alpha, i}+\Gamma^{\alpha}{ }_{i \alpha, j}\right)+\Gamma^{\beta}{ }_{i \alpha} \Gamma^{\alpha}{ }_{j \beta} \\
& -\Gamma^{\beta}{ }_{\alpha \beta} \Gamma^{\alpha}{ }_{i j} \text {, } \\
& \frac{1}{2} \partial_{0}\left(\Gamma_{0 i}^{0}-\Gamma^{m}{ }_{i m}\right)=\frac{1}{2}\left(-2 \Gamma^{m}{ }_{i 0, m}+\Gamma^{\alpha}{ }_{0 \alpha, i}\right) \\
& +\Gamma^{\beta}{ }_{i \alpha} \Gamma^{\alpha}{ }_{0 \beta}-\Gamma^{\beta}{ }_{\alpha \beta} \Gamma^{\alpha}{ }_{0 i} \\
& -\partial_{0} \Gamma^{m}{ }_{0 m}=-\Gamma^{m}{ }_{00, m}+\Gamma^{\beta}{ }_{0 \alpha} \Gamma^{\alpha}{ }_{0 \beta}-\Gamma_{\alpha \beta}^{\beta} \Gamma^{\alpha}{ }_{00} .
\end{aligned}
$$

Equation (2.5a) represents the dynamical equations for $\Gamma^{0}{ }_{i j}$ which are "canonically conjugate" to Eq. (2.4a). Equations (2.5b) and (2.5c) lead to the four differential constraint equations. Thus eliminating $\Gamma^{0}{ }_{i 0}, \Gamma^{m}{ }_{i m}$, and $\Gamma^{m}{ }_{0 m}$ via Eqs. (2.3) and using Eqs. (2.4a) and (2.5a) one obtains four equations not involving any time derivatives. This is the counterpart, in first order form, of the well-known fact that four of the second order Einstein equations contain only first order time derivatives. The four constraint equations, when solved, allow one, in principle, to express four variables from among the twelve quantities $\Gamma_{i j}^{0}, g_{i j}$ in terms of the remaining eight and perhaps $g_{0 \mu}$. This may be symbolized by

$$
h_{\alpha}=h_{\alpha}\left(h_{\beta}, g_{0 \mu}\right), \quad \beta=1,2 \cdots 8 ; \quad \alpha=9,10,11,12 .
$$


Equations (2.5a) and (2.4a) are thus equations giving the time derivatives of $h_{\alpha}$ and $h_{\beta}$, i.e., in the above notation

$$
\begin{aligned}
& \partial_{0} h_{\alpha}=f_{\alpha}, \\
& \partial_{0} h_{\beta}=f_{\beta} .
\end{aligned}
$$

In Eq. (2.7) the $f_{\alpha}$ and $f_{\beta}$ are functions not only of $h_{\alpha}$ and $h_{\beta}$ but also of the four extra variables $g_{0 \mu}$. Note that no time derivatives of $h_{\alpha}$ and $h_{\beta}$ appear in $f_{\alpha}$ or $f_{\beta}$. Equations (2.7a) actually entail no dynamics. Thus if one inserts (2.6) into (2.7a) and eliminates the time derivatives of $h_{\beta}$ by (2.7b), then Eqs. (2.7a) reduce to identities. This is an expression of the Bianchi identities whose effect it is to guarantee that the constraint solutions, Eq. (2.6), are valid at all times. ${ }^{2}$ The remaining eight equations, $(2.7 \mathrm{~b})$, involve the twelve variables $h_{\beta}$ and $g_{0 \mu}$. As will be seen in Sec. IV, there exist choices of $h_{\beta}$ such that no time derivatives of $g_{0 \mu}$ appear in Eq. (2.7b). Hence four of these eight equations can be solved to express $g_{0 \mu}$ as a function of $h_{\beta}$ and $\partial_{0} h_{\beta}$. As will be seen below, the remaining four equations then allow one to express the time derivatives of four particular combinations of the $h_{\beta}$, say $u_{\rho}(\rho=1, \cdots 4)$ in terms of $u_{\rho}, v_{\rho}$, and $\partial_{0} v_{\rho}$, where $v_{\rho}$ represents the other four independent combinations of $h_{\beta}$. Thus Eq. (2.7b) decomposes to read

$$
\begin{aligned}
g_{0 \mu} & =g_{0_{\mu}}\left(u_{\rho}, v_{\rho}, \partial_{0} v_{\rho}\right), \\
\partial_{0} u_{\rho} & =f_{\rho}\left(u_{\rho}, v_{\rho}\right) .
\end{aligned}
$$

Viewing (2.8a) as the equation that determines $g_{0 \mu}$ we see that there exist four quantities, the $v_{\rho}$ in the above notation, which are not determined dynamically. These four quantities are thus the four gauges of the theory and can be chosen as arbitrary functions of the coordinates $x^{\mu}$. Every such choice then represents a coordinate condition and hence determines a coordinate frame. With a particular choice of $v_{\rho}=v_{\rho}\left(x^{\mu}\right)$, the $g_{0 \mu}$ are then uniquely determined and by Eq. (2.4b), one can obtain the four remaining components of the affinity, $\Gamma^{\mu}{ }_{00}$. Equation (2.8b) now represents first order dynamical equations for the four dynamical quantities $u_{\rho}$. It will be seen below that a possible choice of $u_{\rho}$ consists of the four quantities $\left(g_{i j}\right)^{T T}$ and $\left(\left(-g^{00}\right)^{-\frac{1}{2}}\right.$ $\left.\times \Gamma^{0}{ }_{i j}\right)^{T T}$ where the superscript " $T T$ " refers, as in linearized theory, to the transverse traceless part of the quantities:

$$
\partial_{j}\left(A_{i j}\right)^{T T}=0, \quad\left(A_{i i}\right)^{T T}=0 .
$$

Thus the analysis of the structure of the dynamics up to this point is identical with the linearized approximation treated in I. There exists, however, one rather striking dissimilarity between the full theory's dynam-

\footnotetext{
${ }^{2}$ Analogously electromagnetic theory possesses one "Bianchi identity" since it involves only one gauge function. 'Thus, defining $R^{\mu}=F^{\mu \nu}{ }_{\nu} \quad\left(F^{\mu \nu}\right.$ are the electromagnetic field strengths), then $R^{\mu}, \mu \equiv 0$. This guarantees that the $\boldsymbol{\nabla} \cdot \mathbf{E}=0$ constaint equation is preserved in time.
}

ical equations of motion and the linearized ones. This lies in the fact that the gauge transformations of the full theory represent general coordinate transformations. Thus we have that a "dynamical" motion in time can be simulated by a coordinate transformation. This is mirrored in the appearance of the arbitrary gauge functions $v_{\rho}$ in Eq. (2.8b). These equations for the time development of $u_{\rho}$ are only meaningful once one has settled on some specific choice of $v_{\rho}$, i.e., chosen a particular coordinate frame. Once this has been done, one has a unique set of equations that describe the development of the dynamical variables in time in this frame.

Thus, while the gauge functions cancel out in the linearized theory (as in electrodynamics), in the full theory they must be explicitly chosen to define the coordinate frame. The first order gauges are also coordinate transformations but infinitesimal ones which turn out to be determined only in second order theory, as discussed in Sec. 4.

Assuming that a choice has been made for the functions $v_{\rho}$, the four first order equations (2.8b) are not necessarily in canonical Hamiltonian form for two pairs of canonical coordinates. In order to put the equations into canonical form one must choose particular combinations of the $u_{\rho}$ to form two canonical coordinates and their conjugate momenta. The Hamiltonian $H$ governing this system will then depend parametrically on $v_{\rho}(x)$. Thus $H$ will be different in different coordinate systems. (A simple example is given by two coordinate systems differing by a choice of time scale where it is clear that the Hamiltonians in the two systems must differ by a scale factor.) More significantly, for an arbitrary choice of $v_{\rho}, H$ will in general depend explicitly on the time coordinate $x^{0}$ of the frame in question, thus appearing to govern the behavior of a nonconservative system. On the other hand, it is clear that the free gravitational field being described here is an isolated system. For all other isolated physical systems, the coordinate frame is chosen such that energy is conserved. The maintenance of this criterion requires then that one choose a specific coordinate frame (i.e., a specific set of $v_{\rho}$ ) such that in this frame $H$ is independent of time. On physical grounds, such a frame presumably exists and is essentially unique. In this special frame, the Hamiltonian is well defined and represents the physical energy of the free gravitational field. Note that we have hereby introduced the notion of a preferred frame, ${ }^{3}$ the "radiation gauge," to define the physical concept of energy; of course energy can be subsequently discussed in any other frame by making coordinate transformations since the general covariance of the theory has not been lost. The radiation frame thus singled out now supplies

${ }^{3}$ That the introduction of a preferred frame may be necessary to make physical statements in general relativity has previously been suggested by V. Fock [e.g., Revs. Modern Phys. 29, 325 (1957)]. 
one with a coordinate system ${ }^{4}$ which, when compared with the one used in the rest of physics, is in accord with it. It should be noted that the transformation to the coordinate frame where the Hamiltonian is time independent is a coordinate transformation and should not be confused with a canonical transformation of the Hamilton-Jacobi type where one is led to a zero Hamiltonian.

Some of the above ideas will be developed in more detail analytically below. Before doing this, however, we will need to reformulate the equations in terms of quantities having geometrical significance. This will be done in the next section.

\section{GEOMETRICAL FORMULATION}

Due to the purely quadratic nature of the Lagrangian in the linearized theory, the appropriate dynamical variables were simple combinations of the original variables $\mathfrak{g}^{\mu \nu}$ and $\Gamma_{\mu \nu}^{\alpha}$ appearing in the curvature scalar. In the full theory this is not the case; as will be seen below, the fundamental equations simplify greatly and acquire a natural geometrical meaning if combinations of the variables suggested by the elimination of the algebraic constraints are taken.

The Hamiltonian formulation to be reached treats the time development of the dynamical variables referring to a three-dimensional space-like surface which we here choose to be $x^{0}=$ constant. This is seen explicitly in Eq. (2.4a) where only the three-dimensional metric, $g_{i j}$ appears. Thus we adopt the $g_{i j}$ as a preliminary set of dynamical coordinates. The inverse to the three dimensional metric, $\gamma^{i j}$, then obeys the equation

$$
\gamma^{i j} g_{k j}=\delta^{i}{ }_{k} .
$$

It is clear from Eq. (2.5a) that the "canonically conjugate" variables to $g_{i j}$ are related to the $\Gamma_{i j}^{0}$. Instead of using these quantities directly, it is analytically convenient and geometrically more significant to replace them by an equivalent set of variables. ${ }^{5}$ Thus in Eqs. (2.3), the denominators can be eliminated if a $\left(-g^{00}\right)^{-\frac{1}{2}}$ is absorbed into variables possessing a contravariant time index. Thus we define

and

$$
K_{i j}=-N \Gamma_{i j}^{0}, \quad N=\left(-g^{00}\right)^{-\frac{1}{2}}
$$

$$
\zeta^{i}=N g^{0 i} \text {. }
$$

The other components of the four-dimensional metric tensor can then be expressed in terms of these variables:

$$
\begin{gathered}
g^{i j}=\gamma^{i j}-\zeta^{i \zeta^{j}}, \quad g_{00}=-N^{2}\left(1-\zeta^{i} \zeta_{i}\right), \\
g_{0 i}=N \zeta_{i} \equiv \eta_{i} .
\end{gathered}
$$

${ }^{4}$ The form of the coordinate conditions may of course be modified in the presence of matter because the basic condition of time independence now must be imposed on the total Hamiltonian of gravitational field plus matter.

5 The properties of these variables are discussed in detail in a forthcoming paper by C. Misner.
Latin indices here and below are raised and lowered by means of the three-dimensional metric tensors, $g_{i j}$ and $\gamma^{i j}$. Note also that

$$
(-g)^{\frac{1}{2}}=N(3 g)^{\frac{1}{2}}
$$

where $g$ is the determinant of the four-dimensional metric and ${ }^{3} g$ the determinant of the corresponding three-dimensional metric $g_{i j}$. $K_{i j}$ and $\zeta_{i}$ are tensors under three-dimensional coordinate transformations.

In terms of these quantities, Eqs. (2.4a) and (2.5a) become

$$
\partial_{0} g_{i j}=-2 N K_{i j}+\left(N \zeta_{i}\right)_{\mid j}+\left(N \zeta_{j}\right)_{\mid i},
$$

$$
\begin{aligned}
\frac{1}{N} \partial_{0} K_{i j}= & { }^{3} R_{i j}+K K_{i j}-2 K_{i m} K^{m_{j}} \\
& -\frac{1}{N}\left[N_{\mid i j}-N_{\mid i} K_{j m} \zeta^{m}-N_{\mid j} K_{i m} \zeta^{m}\right] \\
& \quad+K_{i m} \zeta^{m}{ }_{\mid j}+K_{j m} \zeta^{m}{ }_{\mid i}+K_{i j \mid m} \zeta^{m} .
\end{aligned}
$$

In Eqs. (3.6) ${ }^{3} R_{i j}$ is the three dimensional contracted curvature formed from $g_{i j}$ and $\gamma^{i j}$. Similarly the subscript symbol " $i$ " represents covariant differentiation with respect to the three-dimensional metric. Equations (3.6) are the dynamical equations for $g_{i j}$ and $K_{i j}$. The differential constraint variables have yet to be eliminated, however.

The defining equations for $\Gamma^{\mu}{ }_{00}$, Eq. (2.4b) become

$$
\begin{aligned}
& \Gamma_{00}^{0}=N^{-1} \partial_{0} N+N_{1 i} \zeta^{i}-N \zeta^{i} \zeta^{j} K_{i j}, \\
& \Gamma_{00}^{i}=N \gamma^{i j} \partial_{0} \zeta_{j}+\frac{1}{2} \gamma^{i j}\left[N^{2}\left(1-\zeta_{m} \zeta^{m}\right)\right]_{, j}-N N_{1 j} \zeta^{i} \zeta^{j} \\
& +N^{2} \zeta^{i} \zeta^{j} \zeta^{k} K_{j k},
\end{aligned}
$$

while the differential constraint equations that replace $(2.5 \mathrm{~b}, \mathrm{c}) \mathrm{read}^{6}$

$$
\begin{aligned}
R_{n n}-\frac{1}{2} g_{n n} R & \equiv \frac{1}{2}\left({ }^{3} R+K^{2}-K_{i j} K^{i j}\right)=0, \\
R_{n i}-\frac{1}{2} g_{n i} R & \equiv-\left(K^{j}{ }_{i}-\delta^{j}{ }_{i} K\right)_{\mid j}=0 .
\end{aligned}
$$

In Eqs. (3.8) the subscript " $n$ " refers to the direction normal to the spacelike surface $x^{0}=$ constant while " $i$ " represents the three directions within the surface. Also $K \equiv \gamma^{i j} K_{i j}$ is the trace of the tensor $K_{i j}$. Finally, the algebraic constraint equations (2.3) become in this notation

$$
\begin{aligned}
& \Gamma^{j}{ }_{i k}={ }^{3} \Gamma^{j}{ }_{i k}+\zeta^{j} K_{i j}, \\
& \Gamma_{0 i}^{0}=N^{-1} N_{\mid i}-K_{i j} \zeta^{j}, \\
& \Gamma_{i 0}^{j}=N\left[-K^{j}+\zeta^{j}{ }_{1 i}+\zeta^{j} K_{i m} \zeta^{m}\right] .
\end{aligned}
$$

In Eq. (3.9a), ${ }^{3} \Gamma^{j}{ }_{i k}$ is the three-space affinity formed from $g_{i j}$ and $\gamma^{i j}$.

The formulation of the theory in the above notation has a direct geometrical significance in terms of the properties of a three-dimensional surface imbedded in a

${ }^{6}$ For a discussion of these equations see, for example, C. W. Misner and J. A. Wheeler, Ann. Phys. (N.Y.) 2, 525 (1957), Eqs. (224), (225). 
four-space. The $K_{i j}$ arise in differential geometry as the components of the so-called second fundamental form. ${ }^{7}$ Thus the $K_{i j}$ represent a measure of the "bending" of the surface with respect to the four-dimensional Riemannian space in the sense that they are a measure of the deviation of the surface from the four-dimensional geodesics connecting two points. The $K_{i j}$ involve information concerning the continuation of the metric off the surface. This can be seen explicitly in Eq. (3.6a) which states that $K_{i j}$ is essentially proportional to the time derivative of $g_{i j}$, as expected for a canonical momentum. Equations (3.8) represent the GaussCodazzi equations ${ }^{7}$ in a geometry restricted by the Einstein equations. These equations clearly involve no time derivatives.

\section{DEFINITION OF ENERGY AND REDUCTION OF NUMBER OF VARIABLES}

We shall now turn to the examination of the meaning of energy in general relativity and to the closely related subject of the nature of the independent degrees of freedom. While in principle this can be analyzed in terms of the equations of motion as discussed in Sec. 2, it is much easier to deal with these questions from the action integral itself. In the notation of the previous section the action integral becomes, by direct substitution into Eq. (2.1),

$$
\begin{aligned}
I=\int d^{4} x \mathcal{L}=\int d^{4} x\left({ }^{3} g\right)^{\frac{1}{2}}\left[-\gamma^{i j} \partial_{0} K_{i j}-\partial_{0} K\right. \\
+N\left({ }^{3} R+K^{2}-K_{i j} K^{i j}\right)-2 \eta^{i}\left(K^{j}{ }_{i}-\delta^{j} K\right)_{\mid j} \\
\left.-2\left(N_{\mid i}-N K_{i j} \zeta^{j}\right)^{\mid i}\right] .
\end{aligned}
$$

In obtaining (4.1) we have explicitly eliminated the algebraic constraint variables by making use of Eqs. (3.9). Due to this elimination, the action integral of Eq. (4.1) yields the differential equations of motion obeyed by the dynamical variables, i.e., Eqs. (3.6) upon varying with respect to $g_{i j}$ and $K_{i j}$, and the constraint equations (3.8) upon varying with respect to $N$ and $\eta^{i}$. These variations simultaneously lead to the generators of canonical transformations $G_{\chi}$ and $G_{x}$. These quantities are

$$
\begin{aligned}
& G_{\chi}=-\int d^{3} r\left[\left({ }^{3} g\right)^{\frac{1}{2}} \gamma^{i j} \delta K_{i j}+\left({ }^{3} g\right)^{\frac{1}{2}} \delta K\right], \\
& G_{x}=\int T^{0 \mu^{\prime}} \delta x_{\mu}\left({ }^{3} g\right)^{\frac{1}{2}} d^{3} r,
\end{aligned}
$$

where $T^{\mu \nu^{\prime}}$ can be obtained by techniques analogous to those of paper I. We do not record it here as we will see shortly that it vanishes.

${ }^{7}$ See, for example, L. P. Eisenhart, Riemannian Geometry (Princeton University Press, Princeton, New Jersey, 1949).
In this form, it is clear that the variables $N$ and $\eta^{i}$ are gauge functions and not dynamical quantities as they do not appear in the "kinematic" part of the Lagrangian (the part containing time derivatives) nor in the generator $G_{x}$. Their only role is that of Lagrange multipliers in the action integral. Thus the Lagrangian now has the form (to within a divergence)

$$
\mathfrak{L}=\sum_{a=1}^{7} \pi_{a} \dot{\phi}_{a}-\mathfrak{H C}^{\prime}(\pi, \phi),
$$

where $\mathfrak{F C}^{\prime}$, the "Hamiltonian" density in the action principle, is given by

$$
\begin{array}{r}
-\mathfrak{H C}^{\prime}=\left[N\left({ }^{3} R+K^{2}-K_{i j} K^{i j}\right)\right. \\
\left.-2 \eta^{i}\left(K^{j}{ }_{i}-\delta^{j}{ }_{i} K\right)_{\mid j}\right]\left({ }^{3} g\right)^{\frac{\hat{R}}{2}} .
\end{array}
$$

From Eq. (4.4) it follows by direct calculation that $P^{0 \prime}=\int d^{3} r \mathcal{C}^{\prime}$ and thus $\mathcal{F C}^{\prime}$ represents the "energy" density. As can be seen from Eqs. (3.8), the function $\mathfrak{F}^{\prime}$ is identically zero since it is a linear combination of the differential constraints. Thus $P^{0 \prime}$ does not generate true time translations in the theory and hence is not the true Hamiltonian. More generally, the complete generator $G_{x}$ vanishes identically for the same reason. In order to see the meaning of this result let us insert the differential constraints into the Lagrangian of Eq. (4.1) just as we previously inserted the algebraic constraints. Leaving aside the irrelevant divergence term, the action reduces down to the simple form

$$
I=\int d^{4} x\left({ }^{3} g\right)^{\frac{1}{2}}\left[-\gamma^{i j} \partial_{0} K_{i j}-\partial_{0} K\right]
$$

Varying this action leads to the expression

$$
\begin{array}{r}
\delta I=\int d^{4} x\left\{-\delta\left[\left({ }^{3} g\right)^{\frac{1}{2}} \gamma^{i j}\right] \partial_{0} K_{i j}+\partial_{0}\left[\left({ }^{3} g\right)^{\frac{1}{2}} \gamma^{i j}\right] \delta K_{i j}\right. \\
-\delta\left[\left({ }^{3} g\right)^{\frac{1}{2}}\right] \partial_{0} K+\partial_{0}\left[\left({ }^{3} g\right)^{\frac{1}{2}}\right] \delta K \\
\left.-\partial_{0}\left[\left({ }^{3} g\right)^{\frac{1}{2}} \gamma^{i j} \delta K_{i j}+\left({ }^{3} g\right)^{\frac{1}{2}} \delta K\right]\right\}
\end{array}
$$

The total time derivative then gives rise to the new generator

$$
G=-\int d^{3} r\left({ }^{3} g\right)^{\frac{1}{2}}\left(\gamma^{i j} \delta K_{i j}+\delta K\right) .
$$

Care must be taken in obtaining the equations of motion from the remaining terms of (4.7). Thus not all the $\gamma^{i j}$ and $K_{i j}$ are independent since we have assumed at this point that the differential constraint variables have been eliminated. In the linearized theory the constraint equations eliminated the four quantities $g^{T}, K_{i}{ }^{T}$, and $K^{T}$ where these variables are related to to $g_{i j}$ and $K_{i j}$ according to the general orthogonal decomposition of a symmetric tensor $f_{i j}=f_{j i}$ :

$$
f_{i j}=f_{i j}{ }^{T T}+f_{i j}{ }^{T}+\frac{1}{2}\left(f_{i}{ }^{T},{ }_{j}+f_{j}{ }^{T}, i\right)+f^{L}{ }_{, i j} \text {. }
$$


In Eq. (4.9), $f_{i j}{ }^{T}$ is defined by

$$
f_{i j}{ }^{T}=\frac{1}{2}\left(\delta_{i j} f^{T}-\frac{1}{\nabla^{2}} f^{T}, i j\right), f_{i j}{ }^{T}, j=0, f_{i i}{ }^{T}=f^{T} .
$$

$f^{T}$ is thus the trace of the transverse part of $f_{i j} . f_{i}^{T}$ is a transverse vector $\left(f_{i}{ }^{T}, i=0\right)$ and $f^{L}$ is a scalar. (Each of the six independent quantities, $f_{i j}{ }^{T T}, f^{T}, f_{i}^{T}$, and $f^{L}$, is uniquely obtainable from $f_{i j}$.) Hence in the linearized case, the dynamical variables are $g_{i j}{ }^{T T}$ and $K_{i j}{ }^{T T}$ while the four quantities $g_{i}^{T}, g^{L}, K^{L}$, are arbitrary gauge functions not determined by the theory. It is easy to see that this result is general and holds in the full theory. Thus the constraint equations again determine $g^{T}, K_{i}{ }^{T}$, and $K^{T}$ in terms of the remaining variables. A simple proof of this is supplied by making a general perturbation expansion in Eqs. (3.8). For example in (3.8a), the linearized part of ${ }^{3} R$, $g_{i i, j j}-g_{i j, i j}$, determines the highest order $g^{T}$ in terms of lower order structures appearing in the non-linear terms and thus to each order, the problem is identical to that of linearized theory. A corresponding result holds for the other three constraints. Similarly Eqs. (3.6) determine the dynamical motion of $\boldsymbol{g}_{i j} \boldsymbol{T} \boldsymbol{T}$ and $K_{i j}{ }^{T T}$ and so these are dynamical variables as again the nonlinear terms in these equations always involve lower order quantities in any given order of perturbation theory. The four quantities, $g_{i}{ }^{T}, g^{L}$ and $K^{L}$ are arbitrary and in fact are an example of the $v_{\rho}$ variables introduced in Sec. 2.8 Thus a specification of these functions represents a statement of the coordinate conditions.

Returning to the question of obtaining the equations of motion from (4.7), one has that the part of $\delta I$ that gives rise to the equations of motion is

$$
\begin{aligned}
\int d^{4} x\left\{-\delta\left[\left({ }^{3} g\right)^{\frac{1}{2}} \gamma^{i j}\right] \partial_{0}\right. & K_{i j}+\partial_{0}\left[\left({ }^{3} g\right)^{\frac{1}{2}} \gamma^{i j}\right] \delta K_{i j} \\
& \left.-\delta\left({ }^{3} g\right)^{\frac{1}{2}} \partial_{0} K+\partial_{0}\left({ }^{3} g\right)^{\frac{1}{2}} \delta K\right\}
\end{aligned}
$$

where in Eq. (4.11) $\gamma^{i j}$ and $K_{i j}$ are now to be viewed as functionals of only the eight variables $g_{i j}{ }^{T T}, K_{i j}{ }^{T T}$, $g_{i}{ }^{T}, g^{L}$, and $K^{L}$. Variation with respect to the dynamical variables produce the four dynamical equations of motion; variation of the remaining (arbitrary) variables gives rise then to the four Bianchi identities of Eqs. (3.6). The remaining four equations of (3.6) of course no longer appear as they are just defining equations for the "Lagrange multipliers" $N$ and $\eta^{i}$ in terms of the remaining variables as in Eq. (2.8a). The $N$ and $\eta^{i}$, being Lagrange multipliers, of course also disappear when the constraints have been eliminated.

Turning next to the generating function, Eq. (4.8), it would appear that there remains no contribution analogous to $G_{x}$ of conventional field theories and hence

8 The twelve equations (3.6) thus divide into the four dynamical equations discussed in the text, four Bianchi identities whose role is to maintain the constraint equations in time and four equations that determine $N$ and $\zeta_{i}$ as functions of $g_{i}{ }^{T}, g^{L}$, and $K^{L}$. This decomposition is identical to the one discussed in Sec. II. no generator of space-time translations. Again, it must be remembered that not all the variables are independent and the insertion of the constraints which must again be done leaves one with only eight variables. Thus the general form of $G$ is

$$
\begin{aligned}
G=\int d^{3} r\left[f^{1}{ }_{i j} \delta g_{i j}{ }^{T T}\right. & +f^{2}{ }_{i j} \delta K_{i j}{ }^{T T} \\
& \left.+f^{3} \delta K^{L}+f^{4} \delta g^{i},{ }_{i}+f^{5}{ }_{i} \delta g_{i}{ }^{T}\right]
\end{aligned}
$$

where the $f^{a}$ are functions of the eight variables. The first terms involving $\delta g^{T T}$ and $\delta K^{T T}$ are of the type that appears in the generating function $G_{x}$. However, they are not yet in the canonical form of $p \delta q-q \delta p$. The remaining four terms still do not have a definite physical meaning. The reason for this lies in that we have not yet chosen a definite coordinate condition; that is, we have not stated what functions of $x^{\mu}$ the $K^{L}, g^{L}, g_{i}{ }^{T}$ are. Thus, if we define four functions $y^{\mu}$ : $y^{0}=-K^{L}, y^{i}=\frac{1}{2} g^{L}{ }_{i}+g_{i}{ }^{T}$, then the coordinate conditions amount to specifying $y^{\mu}$ as given functions of $x^{\nu}$, $y^{\mu}(x)$. The variations in the last four terms can then be replaced by ${ }^{9}$

$$
\delta y^{\mu}=y^{\mu}{ }_{, \nu} \delta x^{\nu},
$$

which gives these four terms the structure $F_{\mu}\left(g^{T T}, K^{T T}, x\right)$ $\times \delta x^{\mu}$. This result is thus of the form $T^{0}{ }_{\mu} \delta x^{\mu}$, i.e., that associated with the generator of space-time translations $G_{\chi}$. We see than that a Hamiltonian or more generally a stress-energy tensor arises only after a set of coordinate conditions have been imposed, although any set of coordinate conditions will do at this stage.

As was mentioned above, though the first part of the generator depends upon dynamical variables, it is not in canonical form. The variables in terms of which $G$ is canonical will in general be functions of $g_{i j}{ }^{T T}, K_{i j}{ }^{T T}$, and $y^{\mu}(x)$. That such a transformation to canonical form can always be made is shown in the Appendix. In terms of the canonical variables, $G$ will be of the form

$$
G=\int d^{3} r\left[g^{c}{ }_{\alpha} \delta K_{\alpha}^{c}+T^{0 \mu} \delta x_{\mu}\right]
$$

where $g_{\alpha}^{c}, K_{\alpha}^{c}(\alpha=1,2)$ are the two canonical pairs replacing the four $g_{i j}^{T T}, K_{i j}{ }^{T T}$. In general, $T^{0 \mu}$ does not represent the physical stress-energy tensor since for an arbitrary choice of the functions $y^{\mu}(x), T^{0 \mu}$ will in general be an explicit function of $x^{\mu}$. On physical grounds one requires, for a closed system, that $T^{0 \mu}$ only depend on $x^{\mu}$ implicitly; this then determines the choice of the coordinate conditions $y^{\mu}(x)$. Thus only for a particular choice of coordinates will $T^{0 \mu}$ be independent of $x^{\mu}$. This particular frame (which we will call the "radiation frame") plays the role of the radiation

${ }^{9}$ More generally, the coordinate conditions may read $y^{\mu}$ $=y^{\mu}\left(x, g^{T T}, K^{T} T^{T}\right)$. In this case $\delta y^{\mu}$ will have extra terms contributing to the first two terms of the generator. This possibility will be discussed in more detail in Sec. V. 
gauge in electrodynamics. Here the quantity

$$
P^{\mu}=\int T^{0 \mu} d^{3} r
$$

represents the physical energy and momentum of the gravitational field.

This situation discussed above in general relatively is formally very similar to the parametric formulation of the Hamilton principle for classical mechanics. ${ }^{10}$ There, one introduces an extra coordinate $q_{n+1}$ to represent the time and a canonical momentum $p_{n+1}$ $=-H$ where $H$ is the Hamiltonian of the original system. The action then reads

$$
I=\int d \tau \sum_{i=1}^{n+1} p_{i} \frac{d q_{i}}{d \tau}
$$

where $\tau$ is an auxiliary parameter and may be arbitrarily chosen. The variation is subject to the constraint

$$
R \equiv p_{n+1}+H=0 .
$$

More generally $R$ can be any function of all the $q_{i}$ and $p_{i}$ such that the equation $R=0$ yields as its solution $p_{n+1}=-H$. This solution may be regarded as the defining expression for the Hamiltonian. Introducing a Lagrange multiplier, $N(\tau)$, the action becomes

$$
I=\int d \tau\left[\sum_{i=1}^{n+1} p_{i} \frac{d q_{i}}{d \tau}-N(\tau) R\left(q_{i}, p_{i}\right)\right]
$$

where now all the $p_{i}, q_{i}$, and $N$ may be varied independently. Equation (4.18) is analogous to Eq. (4.1), $\tau$ playing the role of the coordinates $x^{\mu}$. The generator associated with this action is

$$
G=\sum_{i=1}^{n+1} p_{i} \delta q_{i}-N(\tau) R \delta \tau .
$$

As in Eq. (4.3), the "Hamiltonian" $N R$ vanishes modulo the constraints (which are derived by varying I with respect to $N$ ). Inserting the constraints into (4.19) reduces the generator to

$$
G=\sum_{i=1}^{n} p_{i} \delta q_{i}-H \delta q_{n+1}
$$

Thus the true Hamiltonian arises only when the constraints are utilized. The analog of the coordinate conditions of general relativity consists in specifying $q_{n+1}=q_{n+1}(\tau)$. Then $\delta q_{n+1}=\left(d q_{n+1} / d \tau\right) \delta \tau$. This is possible since the canonical equations do not determine $q_{n+1}$ (just as they do not specify the $y^{\mu}$ defined in the relativity case). Thus, for an arbitrary choice of the function $q_{n+1}(\tau)$, the Hamiltonian generating the translations in

${ }^{10}$ C. Lanczos, The Variational Principles of Mechanics (Toronto University Press, Toronto, 1949). the chosen $\tau$ frame has the form

$$
H d q_{n+1}(\tau) / d \tau,
$$

and is in general $\tau$ dependent even for an initially conservative system. Only with the special choice $q_{n+1}=\tau$ does $\tau$ become the physical time parameter $t$ with a conservative Hamiltonian. Of course other choices are logically consistent (including making $q_{n+1}$ depend on the remaining dynamical variables) but would lead to time parameters which would not be in accord with those usually defined in physics. If, however, $q_{n+1}$ were to depend on the $q_{i}, p_{i}$, it would seem very difficult to retain any physical interpretation of such a choice at least in the quantum theory.

General relativity differs from the simple classical particle problem as follows: in the classical case, one knows the physical parameter $t, a b$ initio, while in general relativity the coordinates are arbitrary to begin with, i.e., we are given a structure of the type (4.18) without any initially given Hamilton's principle type of action. The choice of the "radiation" coordinate condition we have discussed leads to a coordinate independent $T^{0 \mu}$ and hence to conservation laws for a conservative system. This makes our "radiation" frame agree with the coordinates used in the rest of physics and thus our coordinates correctly match on to the ones at infinity where flat-space classical results hold.

It is interesting to formulate the central results of $I$ for the linearized theory in terms of the above analysis. To zeroth order, which is the background flat space without dynamics, $g_{i j}{ }^{T T}$ and $g^{T}$ vanish. Thus if we write $g_{i j}=\frac{1}{2}\left(h_{i, j}+h_{j, i}\right) \equiv \delta_{i j}$, we see that $h_{i}=x_{i}$ in the zeroth approximation. Similarly, it is easy to see that the zeroth order $K_{i j}{ }^{T T}, K^{T}$, and $K_{i}{ }^{T}$ vanish while $K^{L}=-x^{0} .{ }^{11}$ The last follows from an identity that may be obtained from Eq. (3.6), i.e.,

$$
\partial_{0} K=-N_{\mid i}{ }^{i}+N\left(K_{i j} K^{i j}+K_{\mid i} \zeta^{i}\right) .
$$

In zeroth order this reduces to $\partial_{0} K^{L}=-N$. Since $N=\left(-g^{00}\right)^{-\frac{1}{2}}=1$ in the flat-space limit $K^{L}$ must reduce to $-x^{0}$. We thus can write $g_{i j}=\delta_{i j}+h_{i j}$, where $h_{i j}$ is a first order quantity. Expanding the action of Eq. (4.7) (in which the constraints have already been substituted), one obtains as the only nonvanishing terms to quadratic order

$$
I=\int d^{4} x\left[h_{i j}{ }^{T T} \partial_{0} K_{i j}{ }^{T T}-h^{T},{ }_{i i} \partial_{0} K^{L}\right] .
$$

$h^{T}{ }_{, i i}$ is to be obtained by solution of the constraint equation (3.8a); there the linear terms cancel and $h^{T}{ }_{, i i}$ is found to be quadratic in $K_{i j}{ }^{T T}$ and $h_{i j}{ }^{T T}$. Thus $K^{L}$ in Eq. (4.23) can only be zeroth order, i.e., $\partial_{0} K^{(0) L}$ $=-1$. Substituting in the quadratic results for $h^{T}, i$,

\footnotetext{
${ }^{11}$ Of course, $K^{L}, i j$, which is what enters in the affinity, correctly vanishes in the flat-space limit.
} 
one obtains

$$
\begin{aligned}
I=\int d^{4} x\left[h_{i j}{ }^{T T} \partial_{0} K_{i j}{ }^{T T}-\left\{\frac{1}{4}\left(h_{m n}{ }^{T T},{ }_{i}\right)^{2}\right.\right. & \\
& \left.\left.+\left(K_{i j}{ }^{T T}\right)^{2}\right\}\right] .
\end{aligned}
$$

This is, of course, the action for the linearized theory in terms of the canonical variables. ${ }^{12}$ It should perhaps be noted that even though the "Hamiltonian" density $\mathfrak{H C}^{\prime}$ of Eq. (4.5) vanishes rigorously due to the differential constraints, one can still obtain the correct linearized Hamiltonian from $\mathfrak{H}^{\prime}$ by taking its quadratic parts and then inserting the linearized differential constraints. Thus the processes of inserting constraints and linearizing can be performed in either order, and there is no qualitative difference in the structure of the full theory and its perturbation approximations even through the latter do not possess the full coordinate invariance.

\section{DISCUSSION AND CONCLUSIONS}

In the previous sections a general discussion of the structure of gravitational dynamics and the nature of gravitational energy has been given. The initial statement of the theory was expressed in terms of a Lagrangian that depended upon the sixteen variables remaining after the elimination of the algebraic constraints. The stress-energy tensor derived from this Lagrangian vanishes as a consequence of the differential constraint equations and correspondingly the original "Hamiltonian" of the Lagrangian also vanishes. If the constraint variables are then eliminated from the Lagrangian as a whole (by means of the constraint equations) there results a new structure depending upon four dynamical variables and four quantities which remain undetermined. The specification of these arbitrary quantities as functions of the coordinates corresponds to the definition of a coordinate frame. In this form, a new nonvanishing Hamiltonian density arises in the Lagrangian. This Hamiltonian density, however, will represent the physical energy density of the system provided it has no explicit dependence on the coordinates. This physical criterion is necessary in order that the usual conservation laws for a closed system apply here. Thus, the above physical requirement determines a special choice of the four arbitrary quantities and hence implies that one may physically

12 It should be noted that the first order gauge functions are not determined in the linearized theory itself. The first order gauges, however, will enter in the second order dynamical equations and correspondingly, the second order gauges fail to appear there. In general, the $n$th order gauges are determined in the $(n+1)$ st order approximations. That is, the coordinate frame needed to describe the $n$th order theory is determined in the $(n-1)$ st approximation. This is of course consonant with the statement that in the full theory the gauge functions are determined by the condition that the Hamiltonian be coordinate independent. In electrodynamics, on the other hand, the gauge function cancels our rigorously and need not be specified to discuss motion, since coordinates and gauge are independent. use only a special class of coordinate frames to describe the theory.

We have seen that a possible choice of gauge functions are the $y^{\mu}=\left(-K^{L}, \frac{1}{2} g^{L}{ }_{, i}+g^{T}{ }_{i}\right)$. It is clear, however, that one may choose any other four independent functions, $v^{\mu}$, of the $y^{\mu}$ and the canonical variables ${ }^{13}$ (subject only to regularity conditions of kind requiring $v^{\mu}$ to approach $y^{\mu}$ in the linearized limit). In view of this freedom, any coordinate condition can always be stated in the form

$$
v^{\mu}\left[y^{\mu}, g^{c}{ }_{\alpha}, K^{c}{ }_{\alpha}, x^{\nu}\right]=x^{\mu} .
$$

In particular, the specific conservation requirement on the Hamiltonian forces a specific choice of the functions $v^{\mu}$ in Eq. (5.1) and hence, determines $y^{\mu}$. Thus, we saw in the linearized theory that the $y^{\mu}$ themselves were equal to $x^{\mu}$.

Two different coordinate conditions of the type (5.1) (i.e., two different choices of the functions $v^{\mu}$ ) represent the specification of the two different frames. These frames will in general be related by a coordinate transformation depending on the canonical variables. For this case then, at least one of the two frames depends upon the canonical variables. While in the classical theory such a situation is physically undesirable but not logically inconsistent, it presents in the quantum theory certain serious difficulties. The coordinates of the frame depending on the canonical variables would become $q$ numbers, and hence, the meaning of measurements in the theory would become obscure. A further, related, difficulty appears in the quantum theory. There, the set of coordinate transformations that is allowed is more restricted than in the classical case since in general, a $q$-number coordinate transformation will fail to leave the theory even formally invariant. A similar situation holds in quantum electrodynamics where even in such a relatively linear theory only very few $q$-number gauge transformations are allowed.

Let us consider two different coordinate conditions of the type (5.1) in the quantum theory. As discussed above, at least one of the frames must be a $q$-number one, in general. Since only $c$-number coordinates appear to have physical significance, it would seem that the coordinate conditions leading to $q$-number coordinates must be discarded. The question, therefore, arises as to which, if any choice of coordinates is the $c$ number one since the process of finding canonical variables and quantizing does not seem a priori to impose a condition to determine this. In electrodynamics the corresponding question is which gauges are $c$-number gauges. There the decision is made on physical and consistency grounds. One requires that the primary $c$-number gauge is the radiation gauge and the validity of other gauge choices is determined from their relation to this special gauge. At present, in general relativity, consistency arguments cannot yet be made. However,

${ }^{13}$ We should like to thank Dr. J. Anderson for a helpful conversation on this point. 
the physical criterion would seem to be the following: the primary $c$-number frame is the one in which the Hamiltonian is conserved and other coordinate frames are to be admitted if they are related to this frame by a $c$-number coordinate transformation. If one were to define a different class of frames as the allowed $c$-number frames, the theory so defined could not by any allowed recalibration yield a conserved Hamiltonian operator for the closed system being considered. Such a quantum theory would be experimentally distinguishable from the one we are proposing.

Returning to the classical theory, it is instructive to view Eq. (5.1), not as a coordinate condition but as an expression of the fact that the independent variables that the $g_{\alpha}{ }^{c}$ and $K_{\alpha}{ }^{c}$ depend upon are the gauge functions $v^{\mu}$. An analogous situation holds in the parameter formulation of particle mechanics. There, one could have kept $\tau$ arbitrary and considered the correlation embodied in looking at $q_{i}(\tau)=q_{i}\left[\tau\left(q_{n+1}\right)\right] \equiv q_{i}\left(q_{n+1}\right)$ independent of the particular form of $\tau\left(q_{n+1}\right)$. Correspondingly, in general relativity one can consider $g_{\alpha}^{c}$ as depending on $v^{\mu}$ via the relations $g_{\alpha}^{c}(x)=g^{c} \alpha[x(v)]$ $\equiv g_{\alpha}^{c}(v)$. We here treat $v^{\mu}$ as the independent variables and leave no reference to the original arbitrary set, $x^{\mu}$. In particle mechanics, we write $t$ for $q_{n+1}$ to emphasize the physical interpretation whereby energy is conserved and hence the correlation with the usual definition of time is achieved. Similarly, in general relativity, the proper choice is seen to be connected with the energy conservation and asymptotically flatness conditions and in this fashion one makes contact with the coordinates used in the rest of physics. Viewing the $v^{\mu}$ in the role of independent variables emphasizes again that they will have to be $c$ numbers in the corresponding quantum theory.

In the discussions of this paper, we have analyzed in a formal fashion the structure of the dynamics of general relativity. We have assumed throughout that the nonlinear constraint equations can be solved for the appropriate variables. While this, of course, can be done to a perturbation expansion, it is not possible to find closed-form solutions. In electrodynamics, techniques are available within the framework of the action principle which allow a complete discussion of the theory without the explicit elimination of the constraint variables. In a subsequent work, we will discuss similar procedures for general relativity.

\section{APPENDIX}

That a transformation to canonical variables can always be accomplished can be seen by the following analysis. We return to the form of the generator (4.8) before the constraints have been inserted. According to a classical theorem ${ }^{14}$ on Pfaffian forms, any first order differential form, $F$, in $n$ variables can be written as $F=\sum_{i} f_{i} \delta g_{i}+\delta b$, where $i=1,2, \cdots[n / 2]$ and $f_{i}, g_{i}$, and $b$ are functions of the initial variables. For our case, $n=12$ (i.e., there are six $g_{i j}$ and six $K_{i j}$ ). Terms of the type $\delta b$ which will arise in our reduction are total differentials and therefore can be dropped. Insertion of the first constraint reduces $n$ to 11 and so, by the theorem, $F$ can be written as a sum of $[11 / 2]=5$ terms. This form, $\sum_{i=1}^{5} f_{i}{ }^{\prime} \delta g_{i}{ }^{\prime}$, now has 10 variables, $f_{i}^{\prime}$ and $g_{i}{ }^{\prime}$. Proceeding in like fashion with the remaining three constraints, one reduces the structure to two terms, i.e., $p_{1} \delta q_{1}+p_{2} \delta q_{2}$ which is the desired canonical form. The fact that we are dealing with a continuously infinite number of degrees of freedom does not invalidate the proof since the argument holds as well for $12 N$ variables with $4 N$ constraints. The result actually gives the generator in the Hamilton-Jacobi representation since $G$ contains no $T^{0 \mu} \delta x_{\mu}$ type of term. However, once the theorem has been demonstrated in one representation it clearly holds in all others as they can be reached by canonical transformations from this now canonical form. It should be noted that the absence of a $T^{0 \mu}$ term in the Hamilton-Jacobi representation should not be confused with the vanishing of the $T^{0 \mu^{\prime}}$ in Eq. (4.3). The latter vanishes as a consequence of the constraints, the former as a consequence of reducing the variables to the initial Cauchy data. The Hamilton-Jacobi form of the theory itself is not of too great interest; in it the dynamics of the system is in very implicit form. What is of most interest is the Hamiltonian, which represents the true energy of the field and governs the dynamical motion of the variables.

${ }^{14}$ See, for example, J. M. Thomas, Differential Systems (American Mathematical Society Colloquium Publications, 1937), Chap. IV, Vol. XXI, or P. Libermann, Bull. acad. roy. Belg. 39, 846 (1953). 